\title{
UNIVERSIDAD ABIERTA PARA ADULTOS: UN MODELO DE EDUCACIÓN A DISTANCIA EN LA REPÚBLICA DOMINICANA
}

\author{
(THE OPEN UNIVERSITY FOR ADULTS: DISTANCE EDUCATION AT DOMINICAN REPUBLIC)
}

Fernando Hernández

Universidad Abierta para Adultos (República Dominicana)

\section{RESUMEN}

La Universidad Abierta para Adultos, UAPA ubicada en Santiago de los Caballeros en República Dominicana, inicia sus labores docentes y administrativas en el año 1995. En 12 años de existencia ha graduado 5.094 profesionales en las áreas de Psicología, Idiomas, Negocios, Ciencia Jurídicas y Educación.

Esta institución se encuentra organizada bajo una estructura departamental que incluye Escuelas, Departamentos, Institutos y Centros. El gobierno se articula a través de órganos colegiados (Junta de Directores, Consejo Académico y Comisión Consultiva) y órganos unipersonales (Rector, Vicerrectores, Directores de Escuelas, Departamentos, Institutos y Centros).

La acción docente que se desarrolla en la UAPA se fundamenta en el principio de responsabilidad compartida entre los participantes y el facilitador. La metodología empleada es activa y participativa y las técnicas de aprendizaje se basan en la actividad de los que aprenden.

Desde sus inicios ha tratado de favorecer el aprendizaje de sus participantes haciendo uso de metodologías didácticas con aplicaciones de las TICs. En la actualidad la Universidad dispone de su propio portal educativo que funciona en una plataforma Web propia que le sirve de apoyo a los programas no presenciales o virtuales y a la implementación de cursos en líneas de educación permanente.

Palabras clave: UAPA, educación a distancia, participante, facilitador, PASC, PPROFUNDO. 


\title{
F. HERNÁNDEZ
}

Universidad Abierta Para Adultos: Un Modelo de EduCaCión a Distancia en la República Dominicana

\begin{abstract}
The Open University for Adults, UAPA (Universidad Abierta para Adultos), at Santiago, Dominican Republic begins its academic and administrative functions in 1995. During those 12 years of existence, 5094 professionals have obtained degrees in the fields of Psychology, Languages, Business Administration, Law Sciences and Education.

The institution is organized under a departmental structure that includes Schools, Departments, Institutes and Centers. The government of the institution is regulated via collegiate entities (Board of Trustees, Academic Council and Advisory Board) and unitary forms of government (University Director, Deputy Directors, School Directors and Deans, Department Directors, and Center and Institutes Directors).

The academic activities developed at UAPA are based on the principle of shared responsibility between participants and facilitators. The methodology applied is interactive and participatory and the learning techniques are based on the activity of those engaged in the learning process.

Since its inception, UAPA has favored the participant's learning process using didactic methodologies with applications of ICT's. Currently the University has a web-based educational platform to support virtual and distance programming and offerings and to implement continued education on-line courses.
\end{abstract}

Key words: UAPA, distance education, participant, facilitator, PASC, PPROFUNDO.

\section{PERFIL INSTITUCIONAL}

\section{Fundación}

La Universidad Abierta para Adultos UAPA, es una institución privada sin fines de lucro, con personalidad jurídica y patrimonio propio, creada por iniciativa de la Fundación Pro - universidad Abierta para Adultos, Inc., con su domicilio en la ciudad de Santiago de los Caballeros, República Dominicana, cuyo objeto es la Educación Superior a Distancia en sus diferentes modalidades.

El proyecto de creación de la UAPA fue elaborado en 1991 por un grupo de profesionales del país, guiado por su principal ideólogo el Dr. Angel Hernández y presentado el 11 de septiembre de ese año al Consejo Nacional de Educación Superior (CONES), para fines de evaluación y recomendación de aprobación al Poder Ejecutivo. 
En septiembre del año 1994 el CONES conoció y aprobó el informe de evaluación del proyecto UAPA. Con la autorización previa del organismo la Universidad inició sus labores docentes y administrativas en enero de 1995.

El 12 de octubre de 1995 el poder ejecutivo evaluó el Decreto 230-95 mediante el cual se le otorgó personería jurídica y capacidad legal para expedir títulos académicos con igual fuerza, alcance y validez que los expedidos por las instituciones del nivel superior de igual categoría.

\section{Principios Filosóficos y Finalidades}

La filosofía de la Universidad Abierta para Adultos se fundamenta en el desarrollo de una institución de excelencia académica, preocupada por el hombre y sus realizaciones intelectuales y materiales, en condición de valorar la cultura como un patrimonio universal, de aceptar la crítica y ejercerla responsablemente, capaz de innovar, respetuosa del orden público y las libertades del ciudadano. Todo dentro de un espíritu institucional, democrático y participativo, como es el ideal de nuestra sociedad; pero de eficiencia y honestidad en la realización de sus objetivos.

\section{Principios}

El propósito de la Universidad Abierta para Adultos es el desarrollo de facilidades educativas que garanticen igualdad de oportunidades de estudios de nivel superior a todas las personas mayores de 18 años que no hayan podido acceder a los mismos. Enfoques educativos no tradicionales y a distancia, semipresenciales y no presenciales, constituyen la base de esas facilidades, cuyo fundamento teórico descansa en el reconocimiento de que:

- La educación es un derecho de toda persona, y su satisfacción requiere la atención de los poderes públicos y el sector privado.

- Los adultos, independientemente de su condición social, religión, etnia, ideología política, sexo, localización geográfica, y preferencia sexual, tienen la necesidad de desarrollar al máximo sus capacidades personales, en un proceso de aprendizaje permanente que dura toda la vida.

- El derecho a aprender de todo adulto debe ser garantizado a través de formas distintas de enseñanza que reconozcan y propicien la responsabilidad del mismo en su propio aprendizaje. 


\section{Finalidades}

Para brindar educación superior al servicio de la sociedad, la acción de la UAPA está orientada por las finalidades siguientes.

- Garantizar a la población adulta igualdad de oportunidades educativas de nivel superior.

- Incentivar la democratización de acceso a los estudios universitarios en beneficio de todos los sectores de la población.

- Contribuir al establecimiento de lazos efectivos entre la Universidad y el mundo laboral, incorporando la experiencia adquirida como parte integrante de la formación universitaria.

- Contribuir con la sociedad mediante el desarrollo de una conciencia crítica de sus ciudadanos para solucionar los problemas básicos de la misma.

- Fomentar la calidad y excelencia en sus actividades, estableciendo sistemas de planificación, evaluación y control.

- Contribuir a la difusión del conocimiento y la cultura a través de la extensión universitaria.

- Proporcionar formación integral para el ejercicio idóneo de actividades profesionales, a nivel de grado y postgrado.

- Acoger, defender y promover los valores que les son propios tales como: calidad, solidaridad, equidad, cooperación, responsabilidad, innovación y ética.

Misión

Ofrecer educación superior y permanente de calidad a través de una modalidad a distancia que garantice la formación integral de profesionales y líderes capaces de responder a las exigencias de la sociedad del conocimiento.

Apoyando esta adherencia a la excelencia, el consejo de directores, el consejo académico, el rector, los vicerrectores, los directores de escuelas y departamentos, los facilitadores y el personal administrativo y de servicios se comprometen a:

- Desarrollar y poner en práctica metodologías de avanzada en el proceso docente, orientadas a que el participante sea el principal agente de su formación, actuando con responsabilidad, ideas innovadoras y creatividad. 
- Mantener una planta de facilitadores altamente capacitados y actualizados, comprometidos con la institución valorados por la comunidad universitaria y por la sociedad en su conjunto.

- Propiciar la formación integral de los participantes conjugando de manera armónica y flexible las ciencias, las artes, las humanidades y las tecnologías en la vida universitaria.

- Impulsar la vinculación de la Universidad con los sectores representativos de la sociedad, y fortalecer los planes de asistencia social comunitaria.

- Proporcionar un ambiente de respeto a las normas y valores éticos que nos permitan actuar con transparencia en el cumplimiento de nuestras funciones.

- Reconocer el desempeño y los logros en todos los niveles y crear un ambiente profesional que promueva la superación y realización personal así como el orgullo y la cohesión en la institución.

- Formar profesionales calificados en diferentes áreas vinculadas al desarrollo socioeconómico nacional e internacional que sean capaces de identificarse y presentar soluciones a los problemas de su entorno.

- Promover alianzas estratégicas con entidades de filosofías afines a la nuestra en el entendido de que así lograremos fortalecer la calidad, la diversidad en nuestras ofertas curriculares y mantenernos en un alto grado de competitividad.

\section{Visión}

Ser una universidad líder en educación superior a distancia, con prestigio nacional e internacional, vinculada al desarrollo social.

La Universidad Abierta para Adultos se proyecta hacia el futuro como la más importante institución de educación superior a distancia existente en la República Dominicana:

- Forjadora de profesionales, en distintas áreas del saber, comprometidos con el aprendizaje permanente, la búsqueda de la excelencia en el trabajo e identificados con los más altos valores éticos y humanistas.

- Poseedora de un cuerpo de facilitadores altamente calificados, creativos, innovadores y eficientemente productivos, trabajando en un ambiente universitario estimulante y con salarios justos y competitivos. 
- Con un personal administrativo eficiente y especializado, bien remunerado y comprometido con el éxito de la institución.

- Dotada de edificaciones físicas y tecnologías modernas para satisfacer las necesidades de la comunidad universitaria.

- Aliada con instituciones de sólido prestigio internacional en el campo de la educación abierta y a distancia.

- Integrada a diversas redes telemáticas, académicas y científicas para participar de manera activa y permanente en el quehacer universitario nacional e internacional.

- Preparada para contribuir con el desarrollo cualitativo del Sistema Nacional de Educación Superior, Ciencia y Tecnología, aportando propuestas y estrategias para su mejoramiento.

- Ejecutora de proyectos de investigaciones que contribuyan a la búsqueda de soluciones para los problemas de la sociedad dominicana.

- Reconocida socialmente por la pertinencia de sus programas curriculares y por la innovación metodológica y tecnológica en la formación integral de sus participantes.

- Referente de opinión y partícipe del desarrollo económico, social, cultural, científico y tecnológico de la región del Cibao y del país.

\section{Valores y filosofía operativa}

Todas las decisiones académicas y administrativas de la Universidad Abierta para Adultos se basan en valores. Partiendo de esa convicción filosófica la institución asume como valores estratégicos los siguientes: Calidad, Solidaridad, Equidad, Cooperación, Responsabilidad, Innovación, Recursos Humanos y la Ética. 


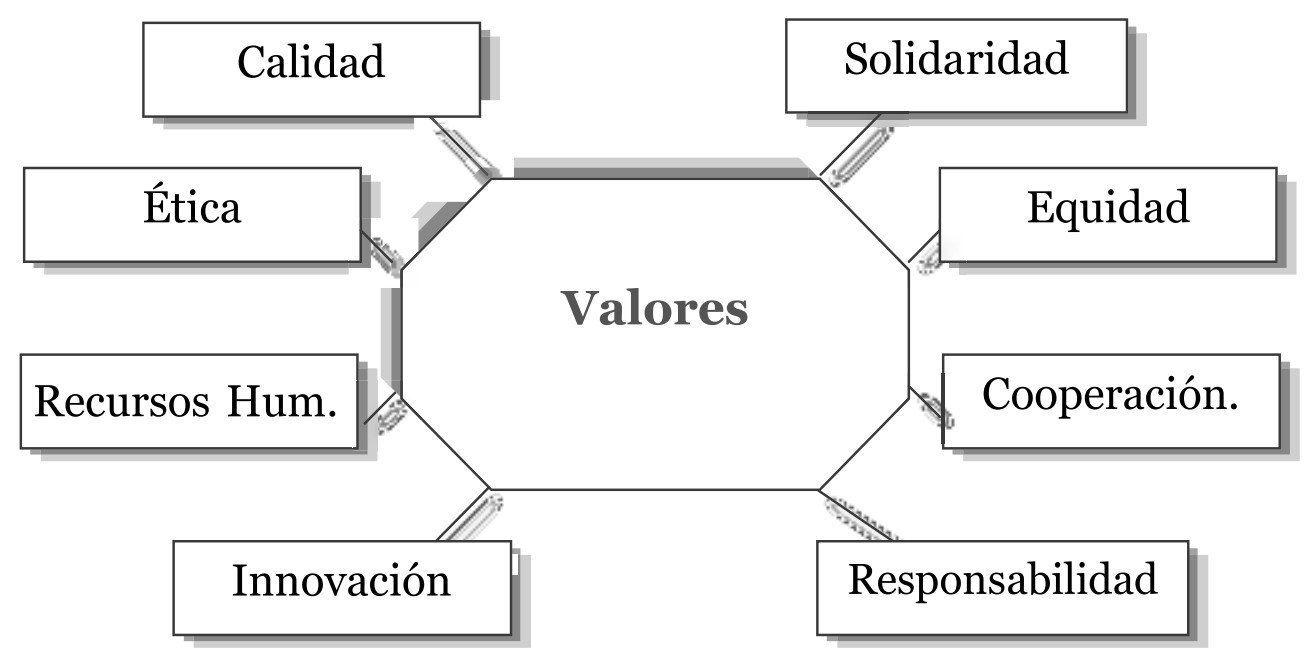

Figura 1 - Valores Estratégicos de la Universidad Abierta para Adultos

Los valores que guían y guiarán la toma de decisiones en la UAPA se encuentran organizados y codificados dentro de su filosofía de operaciones, es decir, la forma como la universidad enfoca su trabajo.

La filosofía operativa de la UAPA se sintetiza en los siguientes principios guías:

Calidad: Concebimos la calidad como la búsqueda de la excelencia a través de la contratación de un personal cualificado, el incentivo a la producción intelectual, la evaluación y planificación constante, y el desarrollo de programas curriculares pertinentes.

Ética: Trabajamos apegados a los principios éticos y morales en las acciones individuales e institucionales.

Recursos Humanos: Reconocemos el valor de la capacidad y la calidad de nuestro personal por lo que nos empeñamos permanentemente en su desarrollo intelectual y en su crecimiento personal. Aceptamos y valoramos la diversidad y el disentimiento. 
Innovación: Porque creemos que innovar es colocarse a tono con el futuro, fomentamos la investigación, experimentamos nuevas ideas y desarrollamos planes creativos que respondan a las necesidades socio-económicas del momento.

Responsabilidad: Los participantes son nuestros principales clientes, existimos por y para ellos, por lo tanto asumimos la responsabilidad de satisfacer sus necesidades y demandas y exigir el cumplimiento de sus deberes, para contribuir con la formación de profesionales íntegros.

Cooperación: Consideramos las relaciones interinstitucionales, las alianzas estratégicas, los intercambios y la cooperación académica, científica y cultural como vías a seguir para enriquecer nuestros recursos y servicios académicos.

Solidaridad: Porque somos sensibles a la realidad económica, social y cultural del país, valoramos y participamos de manera proactiva y solidaria en el desarrollo de programas y actividades orientadas a satisfacer necesidades de las comunidades en que estamos inmersos.

Equidad: Para asegurar la sana convivencia y la cohesión de la comunidad universitaria, procedemos con imparcialidad, integridad y justicia en la solución de conflictos, el acceso a oportunidades que conducen a la superación y en el reconocimiento de méritos, en un marco de igualdad y respetando las normativas.

\section{Estructura Organizacional}

\section{Estructura Académica}

La Universidad Abierta para Adultos se encuentra articulada bajo una estructura académica de tipo departamental que incluye: Escuelas, Departamentos, Institutos y Centros.

Actualmente funcionan las siguientes Escuelas:

- Educación

- Idiomas

- Psicología

- Ciencias Jurídicas y Políticas

- Ciencia y Tecnología

- Turismo 
- Negocios

- Postgrado

\section{Órganos de Gobierno}

El gobierno, representación y administración de la UAPA se articula a través de:

- Órganos Colegiados: Junta de Directores, Consejo Académico y Comisión Consultiva.

- Órganos Unipersonales: Rector, Vicerrectores, Directores de Escuelas, de Departamento, de Recintos, de Institutos, de Centros y de Programas.

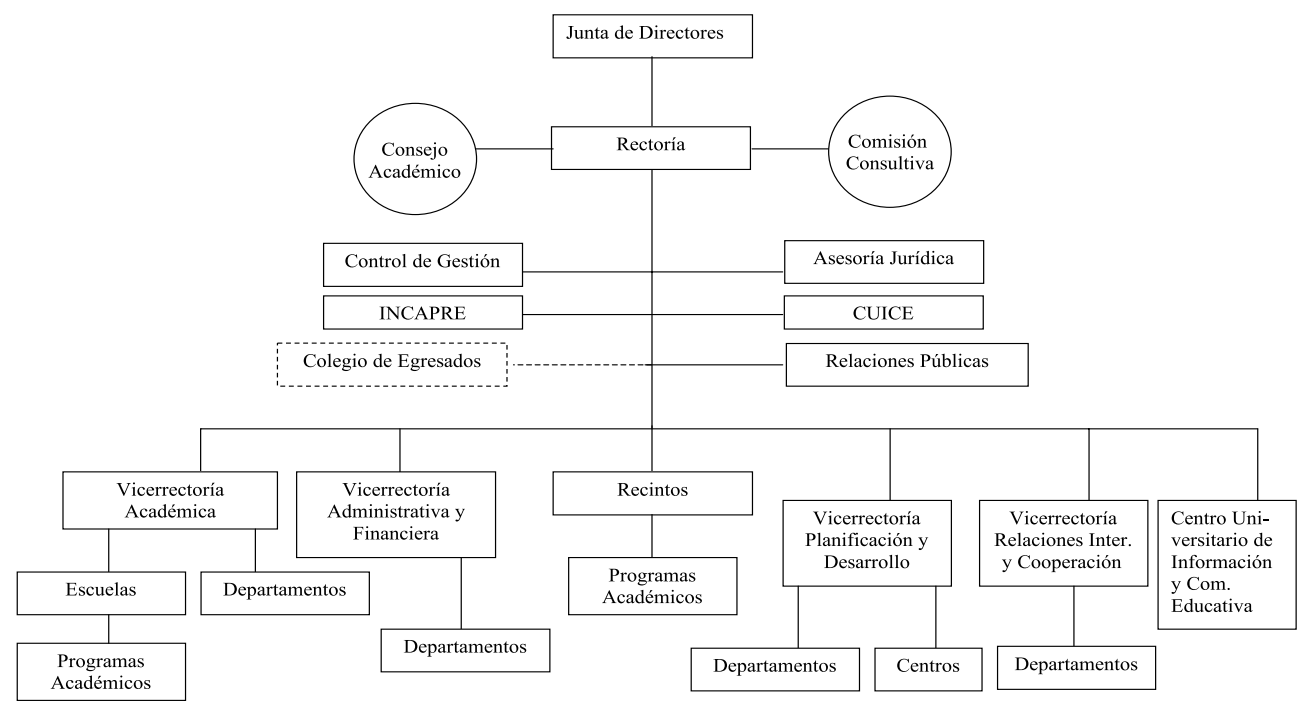

Figura 2 - Organigrama general

\section{Ámbito de Influencia y Población a la que se dirige}

\section{Ámbito Geográfico}

Los Centros o Recintos de docencia de la UAPA se encuentran en tres provincias de la República Dominicana, aunque su influencia se extiende a todo el territorio nacional y ya cuenta con un modesto número de participantes en Estados Unidos. 
La Rectoría se encuentra en Santiago de los Caballeros en la región del Cibao Oriental (norte del país). El Recinto Cibao Oriental en el municipio de Nagua, provincia María Trinidad Sánchez, en la región Nordeste, y el recinto Santo Domingo Oriental que funciona en el municipio de Santo Domingo Este, en la provincia de Santo Domingo, capital de la República.

\section{Población Estudiantil}

La población estudiantil de la UAPA es un grupo heterogéneo de adultos, que van desde jóvenes adultos hasta adultos mayores, predominando en esta población el adulto-joven, de distintos estratos sociales, diferentes niveles de ingresos económicos y con formación académica desde bachilleres recién salidos de la Educación Media, adultos que tienen muchos años que concluyeron estos estudios, hasta personas con maestría que quieren realizar otra carrera de interés o por requerimientos de cambios laborales.

En el artículo 2 de sus Estatutos, la UAPA establece que, con la finalidad de garantizar igualdad de oportunidades de estudio de nivel superior, los destinatarios de su actividad educativa son todas las personas mayores de 18 años que por una u otra causa no han podido acceder a este tipo de educación.

La UAPA tiene dentro de su población estudiantil a jóvenes de 18 años partiendo del hecho de que todos los que tienen 18 años son adultos legalmente. A esta edad se adquiere la responsabilidad principal de todo hombre: ser ciudadano. La capacidad de elegir y ser elegido como gobernante y participación en la vida cívica, cultural, judicial y política con responsabilidad propia. Por eso, también pueden estudiar por su cuenta y la experiencia que hemos tenido nos ha dado la razón.

Actualmente la institución tiene una población de 5.485 participantes.

\begin{tabular}{|l|c|c|}
\hline \multicolumn{1}{|c|}{ Escuela } & Total & Porcentaje \\
\hline Psicología & 849 & $15,48 \%$ \\
\hline Idiomas & 369 & $6,73 \%$ \\
\hline Negocios & 2.049 & $37,36 \%$ \\
\hline Ciencias Jurídicas y Políticas & 1.479 & $26,96 \%$ \\
\hline Ciencia y Tecnología & 83 & $1,51 \%$ \\
\hline Educación & 490 & $8,93 \%$ \\
\hline
\end{tabular}




\begin{tabular}{|l|c|c|}
\hline Postgrado & 166 & $3,03 \%$ \\
\hline Total & $\mathbf{5 . 4 8 5}$ & $\mathbf{1 0 0 \%}$ \\
\hline
\end{tabular}

Tabla 1 - Participantes activos por Escuela (Departamento de Análisis y Prospección, UAPA)

\section{DOCENCIA}

\section{Organización de la Docencia}

La labor docente de la UAPA se orienta a que los participantes adquieran una formación integral que les permita competir favorablemente en el mercado laboral, servir a la sociedad y contribuir a la solución de sus problemas.

Los procesos de enseñanza-aprendizaje se organizan en planes y programas de estudios conducentes a grados académicos y títulos profesionales que se podrán impartir en forma permanente, temporal o eventual, bajo la modalidad educativa a distancia sea semi-presencial, no presencial o virtual.

\section{Metodología}

La Universidad Abierta para Adultos fundamenta su acción en el principio de responsabilidad compartida entre participante y facilitador en el proceso de aprendizaje.

En el plano operativo, esto se traduce en un alto grado de actividades que el participante realiza con la sola orientación del facilitador, generando un proceso educativo en el cual se reconocen las diferencias individuales; tanto en lo relativo a las aptitudes como a la situación social y laboral de los participantes; dando lugar a una oferta curricular flexible en la organización de los horarios de docencia. La metodología empleada es activa, participativa y democrática. Las técnicas de aprendizaje se basan en la actividad de los que aprenden.

Dentro de este contexto, la Universidad prioriza aquellas técnicas de aprendizaje que se fundamentan en los principios de aprenden haciendo y aprender a aprender, dentro de los cuales cabe citar: la investigación, los talleres, seminarios, exposiciones. 
La enseñanza es principalmente semipresencial, lo que implica que una parte significativa del trabajo académico se realiza en el hogar. Dentro de este contexto, todas las técnicas de enseñanza, que se centran en la labor continua del participante en aprender, serán las vías utilizadas, en interés de ofrecerle todas las posibilidades de desarrollo personal y social.

Cada cuatrimestre los educandos seleccionan un bloque de asignaturas, las que se imparten dos por bimestre. Durante el desarrollo de cada asignatura, el grupo de participantes se reunirá con el facilitador una vez por semana (8 en el bimestre). En estos encuentros el facilitador presentará el programa, se distribuirán las actividades a grupos de participantes y se discutirán los contenidos básicos de los programas y las actividades de los módulos instruccionales.

\section{Medios Didácticos}

Actualmente el medio más importante parallevar a cabo el proceso del enseñanzaaprendizaje es el material impreso, ya que más del 95\% de los participantes están inscritos en programas semi-presenciales.

Se utilizan tanto libros preparados para el autoestudio como textos convencionales de mercado los cuales se acompañan con guías didácticas.

Para completar la labor de aprendizaje, tanto facilitadores como participantes se auxilian de recursos didácticos y tecnológicos, tales como: Mapas, Proyectores de vistas fijas, Retroproyector, Televisión, Internet, proyectores de multimedia, etc.

\section{Uso de la TIC's}

Las tecnologías de la información y las comunicación, TIC's, han modificado profundamente los procesos académicos y administrativos de las Instituciones de Educación Superior.

La UAPA desde sus inicios ha tratado de favorecer el aprendizaje de sus participantes haciendo uso de metodologías didácticas con aplicaciones de las TIC's.

En la actualidad la Universidad dispone de su propio Portal Educativo que funciona en una plataforma Web propia diseñada en base a los requerimientos 
institucionales, que sirve de apoyo al programa de formación no presencial y a la implementación de cursos en línea de educación permanente.

También la institución cuenta con facilidades de videoconferencia y modernos laboratorios de informática con acceso a la INTERNET. Todos los servicios académicos y administrativos están computarizados y se actualiza su tecnología periódicamente.

Para lograr el desarrollo de su política de información y comunicación la UAPA creó el Centro Universitario de Información y Comunicación Educativa, CUICE, el cual sustituirá a partir de febrero del 2007 su actual Departamento de Tecnología de la Información y la Comunicación.

El CUICE surge con el propósito de: "contribuir a la misión y planes de la Universidad Abierta para Adultos promoviendo y facilitando el uso de las nuevas tecnologías de la información y la comunicación como recurso de apoyo didáctico y de gestión para mejorar la eficiencia y eficacia de los procesos académicos y administrativos de la institución”. Tiene los objetivos siguientes:

- Fomentar el uso y difusión de las nuevas tecnologías de la información y la comunicación aplicadas a la educación a distancia.

- Contribuir al mejoramiento de la eficiencia y eficacia de los procesos académicos y servicios administrativos mediante el uso de las tecnologías como recurso de enseñanza y de gestión administrativa.

- Desarrollar y gestionar el Portal Educativo de la Universidad.

- Diseñar y producir materiales educativos de multimedia que faciliten el proceso de enseñanza-aprendizaje a distancia en sus distintas modalidades.

La estructura organizativa del CUICE esta conformada por una Dirección Ejecutiva de la cual dependen las unidades operativas. Se contemplan las unidades siguientes:

- Diseño y Producción Multimedia

- Informática Interna

- Soporte Web y Educación en Línea

- Servicios de Audiovisuales 


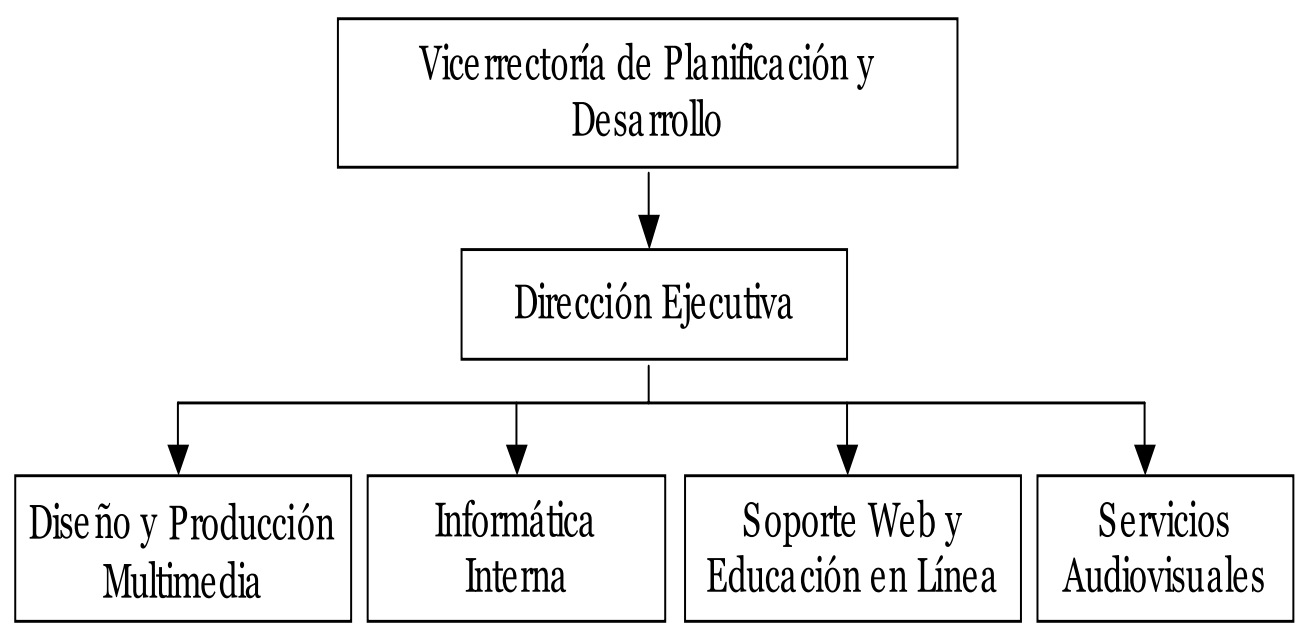

Figura 2 - Organigrama del CUICE

La Unidad de Diseño y Producción de Multimedia está orientada a diseñar y editar los diferentes formatos de elaboración de materiales como son video, audio e imágenes, que se utilizan en la enseñanza. Además se encarga de darle formato a los materiales multimedia para ser colgados en la plataforma Web.

La unidad de Informática Interna es la encargada de gestionar el buen funcionamiento del Sistema de Información de todos los equipos y redes informáticas de la Universidad. Encargándose de gestionar las necesidades de equipos y software informáticos de la Institución en coordinación con la Dirección del CUICE.

La unidad de Soporte Web y Educación en Línea, es la encargada de velar por el buen funcionamiento del Portal de UAPA, actualizar todas las informaciones, distribuir la mensajería electrónica en los diferentes departamentos, colgar los materiales didácticos y cursos en líneas en la plataforma Web.

También es la encargada de dar soporte Web y académico a todas las actividades desarrolladas por los facilitadores en la plataforma Web y en los laboratorios.

La unidad de Servicios Audiovisuales es la encargada de administrar y garantizar el buen funcionamiento de todos los equipos y materiales audiovisuales, los salones y equipos de videoconferencia de la UAPA. También tiene el control y registro de informaciones sobre los préstamos realizados a facilitadores y participantes de la Institución. 


\section{Formación de los Docentes}

\section{El docente en la UAPA}

En la Universidad Abierta para Adultos, por su carácter de institución a distancia, el docente recibe el nombre de facilitador, que es el profesional cuya labor en el seno de la institución consiste en coordinar, guiar y evaluar el proceso de aprendizaje de una asignatura determinada, producir materiales didácticos y guías de estudios, así como participar de los proyectos de investigación y de extensión que se lleven a cabo.

Actualmente la UAPA cuenta con una planta de 197 facilitadores de los cuales $85 \%$ (164) tiene formación a nivel de post-grado y $15 \%$ (28) tiene nivel de licenciatura (tabla 2).

\begin{tabular}{|l|c|c|c|c|c|c|c|c|c|}
\hline \multicolumn{1}{|c|}{ Escuela } & Total & \multicolumn{7}{|c|}{ Nivel Académico y Porcentaje } \\
\hline & & Dotorado & $\%$ & Mlaestría & $\%$ & Especialidad & $\%$ & Licenciatura & \% \\
\hline Ciclo Básico & 37 & - & - & 25 & 68 & 07 & 19 & 05 & 14 \\
\hline Educación & 21 & - & - & 15 & 71 & 06 & 29 & - & - \\
\hline Psicología & 33 & - & - & 23 & 70 & 05 & 15 & 05 & 15 \\
\hline Idiomas & 17 & - & - & 05 & 29 & 01 & 6 & 11 & 65 \\
\hline Negocios & & - & - & 19 & 90 & 02 & 10 & - & - \\
\hline Ciencias Jurídicas y Políticas & 29 & 02 & 7 & 18 & 62 & 04 & 14 & 05 & 17 \\
\hline Ciencia y Tecnología & 10 & - & - & 05 & 50 & 03 & 30 & 02 & 20 \\
\hline Postgrado & 24 & 04 & 17 & 14 & 58 & 06 & 25 & - & - \\
\hline Total & $\mathbf{1 9 2}$ & $\mathbf{0 6}$ & $\mathbf{3}$ & $\mathbf{1 2 4}$ & $\mathbf{6 7}$ & $\mathbf{3 4}$ & $\mathbf{1 6}$ & $\mathbf{2 8}$ & $\mathbf{1 5}$ \\
\hline
\end{tabular}

Tabla 2 - Niveles de Formación Académica del Cuerpo Docente de la UAPA según Escuela y Ciclo Básico, 2006 (Departamento de Análisis y Prospección, UAPA)

\section{Programa de Profesionalización de la Función Docente}

Compartiendo la preocupación por mejorar la calidad de la educación superior a distancia, la UAPA creó en el año 2005 el Programa de Profesionalización de la Función Docente, PPROFUNDO, con el objetivo general de: 
Desarrollar estrategias y acciones orientadas a la profesionalización y revalorización de la función docente que contemplen intervenciones articuladas en la formación, las condiciones de trabajo y el sistema de recompensas, para garantizar la adquisición del perfil y competencias que deben tener los facilitadores para mejorar su eficiencia y eficacia y así acceder a logros educativos de mayor calidad y pertinencia coherentes con la misión y visión institucional.

Son objetivos específicos del PPROFUNDO los siguientes:

- Ofrecer a los facilitadores oportunidades para realizar estudios a nivel de postgrado en IES nacionales e internacionales.

- Fortalecer las competencias y habilidades de los facilitadores mediante acciones pertinentes de capacitación y actualización de los facilitadores activos.

- Impulsar la calidad y pertinencia de la capacitación y actualización de los facilitadores activos.

- Realizar diagnósticos que permitan conocer las necesidades, intereses y aspiraciones de los facilitadores.

- Diseñar y ejecutar proyectos de desarrollo profesional dirigidos al personal docente y a los directivos de la Institución.

- Articular un proyecto de becas nacionales e internacionales orientado a contribuir con el proceso de desarrollo profesional de los facilitadores y directivos de la UAPA.

- Retroalimentar las actividades de apoyo académico que actualmente se realizan en la Universidad a favor de los facilitadores, para que se conviertan en acciones operativas del proceso de desarrollo profesional de los docentes.

- Promover la realización de convenios y acuerdos de colaboración e intercambio académico con instituciones de educación superior, de investigación, asociaciones profesionales y $\mathrm{ONG}^{\prime}$ s para ofrecer actividades innovadoras vinculadas al desarrollo profesional de los facilitadores y directivos de la Universidad.

- Establecer, en coordinación con el Departamento de Evaluación Institucional y del Docente, un sistema de evaluación y seguimiento del impacto que tengan las acciones de desarrollo profesional de los facilitadores y directivos en la elevación de la calidad de los programas curriculares y de los servicios que se ofrecen a la comunidad universitaria.

- Diseñar y manejar un sistema de información y difusión amplio y oportuno de las distintas actividades que se planifiquen y ejecuten. 
- Promover el establecimiento de mecanismos de estímulo y reconocimiento al rendimiento y a la superación de los facilitadores.

Para lograr la profesionalización de la función docente en la Universidad Abierta para Adultos en el marco del desarrollo del presente programa se tendrán en cuenta requisitos vinculados con aspectos académicos, las condiciones de trabajo y el sistema de recompensas.

Entre los aspectos académicos se destacan:

- La formación a nivel de postgrado de los facilitadores.

- La capacitación y el perfeccionamiento (formación continua).

- La formación de formadores.

- La evaluación.

Las condiciones de trabajo incluyen tres aspectos básicos:

- La carrera docente.

- Remuneración laboral.

- Infraestructura y equipamiento didáctico.

El sistema de recompensas incluye estímulos materiales y reconocimientos simbólicos. En el marco de este trabajo sólo se hace referencia a los aspectos relacionados con la formación a nivel de postgrado y la capacitación y perfeccionamiento de los facilitadores.

\section{Formación a nivel de postgrado}

A través del PPROFUNDO la Universidad pretende que en un período no mayor a cinco (5) años a partir del 2005, el 100\% de los facilitadores alcancen titulación a nivel de postgrado, privilegiando los estudios de maestrías y doctorales, con el fin de acceder a mejores logros educativos en los diferentes programas curriculares que se ofertan a la sociedad.

Formación de los facilitadores de nuevo ingreso

Para ingresar como facilitador a la UAPA a partir de la puesta en ejecución del PPROFUNDO se exige una titulación a nivel de postgrado, preferentemente maestría 
y/o doctorado, con las excepciones que establecen el Reglamento de Facilitadores y Carrera Docente y los Estatutos de la Institución

Formación de los facilitadores en Servicio

El PPROFUNDO oferta opciones de estudios de postgrado a los facilitadores a nivel de:

- Especialidad

- Maestría

- Doctorado

Los facilitadores tienen acceso a estudios de postgrado a través de dos opciones básicas:

Opción A: Las áreas de formación responden a prioridades institucionales, las cuales tienen un impacto en los procesos académicos y/o de gestión de la Universidad.

Las áreas y/o programas curriculares correspondientes a la opción A aparecerán establecidas en el Plan Estratégico Quinquenal de la UAPA y en los planes operativos de las diferentes Escuelas y Departamentos. Bajo esta opción la institución en que se realizan los estudios, nacional o internacional, la determina el PPROFUNDO en coordinación con la Escuela y/o Departamento a que pertenezca el interesado. En estos casos la Universidad asume entre 70\% a 100\% de los costos del programa.

Opción B: Responde a programas y áreas de formación de interés para el facilitador. Aquí el interesado elije el programa curricular y la institución que le agrade. Si la Universidad elegida es la UAPA, se le otorga una beca parcial que cubre el 50\% del costo del programa.

\section{Capacitación y perfeccionamiento}

El PPROFUNDO concibe la capacitación y el perfeccionamiento como un proceso permanente inherente al ejercicio profesional de los facilitadores y directivos de la Universidad.

A través de la capacitación y el perfeccionamiento se persigue. 
- Fortalecer la institución elevando el nivel de sus facilitadores y directivos.

- Establecer mecanismos para el intercambio de informaciones sobre las innovaciones en distintas áreas.

- Facilitar las instancias de aprendizaje en talleres y en grupo de facilitadores.

- Analizar las necesidades pedagógicas de cada Escuela y Departamento de la institución.

- Estimular la participación de todos los niveles de conducción para lograr el perfil profesional de los docentes.

El perfeccionamiento docente debe ofrecer oportunidades flexibles para estimular:

- El acceso de todos los facilitadores, asociados a un plan de incentivos.

- La reflexión sobre la función docente como exigencia de carácter profesional.

- La recreación de prácticas pedagógicas modernas.

- El desarrollo de distintos grupos y modalidades de participación de los facilitadores: talleres de educadores organizados por escuelas y departamentos, red de docentes por disciplinas, grupos de investigación.

Este proceso de formación permanente estará orientado a promover en los facilitadores asistentes determinados perfiles profesionales asociados con la autonomía profesional y la creatividad.

La oferta de formación continua estará conformada por:

- Cursos de larga duración

- Cursos cortos

- Pasantías

- Seminarios

- Talleres

- Foros

- Conferencias

Además de la dirección del PPROFUNDO, las Escuelas, los Departamentos y grupos de facilitadores podrán convocar a estos tipos de eventos. 


\section{Áreas de formación permanente}

Las actividades permanentes de capacitación y actualización que ejecuta el PPROFUNDO se desarrollan en distintas áreas del saber dependiendo de las prioridades de la Universidad y según el tipo de beneficiario (facilitador y/o directivo) y el tiempo en servicio. En todo caso estas actividades están orientadas a que los docentes y directivos adquieran las competencias y el perfil profesional que deben tener los docentes en la sociedad de las próximas décadas.

\begin{tabular}{|l|l|}
\hline \multicolumn{1}{|c|}{ CICLO - NIVEL } & \multicolumn{1}{|c|}{ ÁREA TEMÁTICA } \\
\hline $\begin{array}{l}\text { Facilitadores de nuevo ingreso hasta } \\
\text { segundo año en servicio }\end{array}$ & $\begin{array}{l}\text { Educación Superior a Distancia } \\
\text { Tecnología Educativa } \\
\text { Educación en Valores } \\
\text { Estrategias de Intervención Didáctica }\end{array}$ \\
\hline Facilitadores de 3 hasta 5 años en servicios. & $\begin{array}{l}\text { La disciplina según el título de base } \\
\text { Tecnología Educativa } \\
\text { Metodología de la Investigación } \\
\text { Gestión Institucional }\end{array}$ \\
\hline $\begin{array}{l}\text { Facilitadores de } 6 \text { años en adelante en } \\
\text { servicios }\end{array}$ & $\begin{array}{l}\text { La disciplina según el título de base } \\
\text { Gestión Institucional } \\
\text { Tecnología Educativa } \\
\text { Interés propio del facilitador }\end{array}$ \\
\hline Directivos & $\begin{array}{l}\text { Gestión Curricular } \\
\text { Gestión Institucional }\end{array}$ \\
\hline
\end{tabular}

Tabla 3 - Área de formación según ciclo y/o nivel del beneficiado.

\section{Oferta curricular de la UAPA}

La oferta curricular de la UAPA se concentra fundamentalmente en los estudios reglados, siendo el 90\% de su oferta a nivel de Licenciatura y sólo un 10\% de postgrado (especialidad y maestría).

También mantiene una constante oferta de programas de educación permanente de corta y mediana duración (diplomados, talleres, seminarios, entre otros). 
Universidad Abierta para Adultos: Un Modelo de Educación a Distancia en la República Dominicana

\begin{tabular}{|c|c|c|}
\hline Carreras y Menciones & $\mathbf{N}^{\circ}$ de créditos & $\begin{array}{l}\text { Duración en } \\
\text { cuatrimestre }\end{array}$ \\
\hline Derecho & 247 & 14 \\
\hline \multirow{4}{*}{\begin{tabular}{cl}
\multicolumn{2}{l}{ Educación: } \\
• & Básica \\
- & Inicial \\
• & Ciencias Sociales \\
- & Didáctica de \\
& las Lenguas \\
& Extranjeras (Inglés \\
& y Francés)
\end{tabular}} & 212 & 13 \\
\hline & 208 & 13 \\
\hline & 208 & 13 \\
\hline & 220 & 13 \\
\hline \multirow{4}{*}{$\begin{array}{cl}\text { Psicología: } \\
\text { • } & \text { General } \\
\text { • } & \text { Industrial } \\
\text { • } & \text { Clínica } \\
\text { - } & \text { Educativa }\end{array}$} & 225 & 13 \\
\hline & 202 & 12 \\
\hline & 224 & 13 \\
\hline & 229 & 13 \\
\hline $\begin{array}{l}\text { Administración de } \\
\text { Empresas }\end{array}$ & 209 & 12 \\
\hline Mercadeo & 208 & 12 \\
\hline Contabilidad Empresarial & 241 & 13 \\
\hline $\begin{array}{l}\text { Administración de } \\
\text { Empresas Turísticas }\end{array}$ & 232 & 13 \\
\hline $\begin{array}{l}\text { Lenguas Modernas: } \\
\text { Turismo }\end{array}$ & 210 & 12 \\
\hline Informática Gerencial & 195 & 12 \\
\hline
\end{tabular}

Tabla 4 - Carreras ofrecidas por la UAPA a nivel de Licenciatura 2006

\begin{tabular}{|c|c|c|}
\hline Programa y Nivel & $\mathbf{N}^{\circ}$ de Crédito & $\begin{array}{c}\text { Duración en } \\
\text { Cuatrimestre }\end{array}$ \\
\hline \multirow{4}{*}{$\begin{array}{cl}\text { Maestría en: } \\
\text { • } & \text { Mercadeo } \\
& \text { Gerencial } \\
\text { - } & \text { Mercadeo Político } \\
\text { - } & \text { Gestión de Centros } \\
& \text { Educativos } \\
\text { - } & \text { Legislación de } \\
& \text { Tierra }\end{array}$} & 58 & 6 \\
\hline & 58 & 6 \\
\hline & 57 & 6 \\
\hline & 62 & 7 \\
\hline
\end{tabular}




\begin{tabular}{|c|c|c|}
\hline \multirow{4}{*}{$\begin{aligned} & \text { Especialidad en: } \\
& \text { - } \text { Gestión de Centros } \\
& \text { Educativos } \\
& \text { - } \text { Gerencias de } \\
& \text { Recursos Humanos } \\
& \text { - } \text { Mercadeo } \\
& \text { Gerencial } \\
& \text { - } \text { Mercadeo Político }\end{aligned}$} & 30 & 4 \\
\hline & 47 & 5 \\
\hline & 37 & 3 \\
\hline & 37 & 3 \\
\hline
\end{tabular}

Tabla 5 - Programación académica de postgrado ofrecida por la UAPA 2006

\section{Formación de Profesionales}

La labor docente de la UAPA se orienta a la formación de profesionales integrales caracterizados por sus conocimientos, capacitación y valores. Profesionales bien informados, eficientes en su desempeño laboral y comprometido con su país, la región y el servicio comunitario.

En sus doce (12) años de existencia la Universidad ha graduado un total de 5,094 profesionales que desempeñan sus labores en todo el territorio nacional.

\begin{tabular}{|l|c|c|}
\hline \multicolumn{1}{|c|}{ Escuela } & Total & \% \\
\hline Psicología & 263 & $5,16 \%$ \\
\hline Idiomas & 154 & $3,02 \%$ \\
\hline Negocios & 1.107 & $21,73 \%$ \\
\hline $\begin{array}{l}\text { Ciencias Jurídicas y } \\
\text { Políticas }\end{array}$ & 1.358 & $26,66 \%$ \\
\hline Educación & 1.907 & $37,44 \%$ \\
\hline Postgrado & 305 & $5,99 \%$ \\
\hline \multicolumn{1}{|c|}{ Total } & $\mathbf{5 . 0 9 4}$ & $\mathbf{1 0 0 \%}$ \\
\hline
\end{tabular}

Tabla 6 - Egresados por Escuela en la UAPA hasta 2006 (Departamento de Análisis y Prospección, UAPA) 


\section{INVESTIGACIÓN y PUBLICACIONES}

\section{Investigación}

La función de investigación en la UAPA está integrada por el conjunto de procesos de indagación científica y búsqueda del conocimiento que realicen los miembros de la comunidad universitaria, principalmente los facilitadores (docentes) y los participantes (alumnos). Tales procesos contribuyen a la formación integral de los participantes, al desarrollo de una cultura investigadora en la institución y a la solución de problemas comunitarios.

Las líneas de investigación están orientadas a establecer vínculos con la docencia para ofrecer resultados que elevan su calidad; así como con la extensión universitaria para que de sus aportes se beneficien las comunidades de la región y del país.

En la actualidad la institución está tratando de desarrollar esa función universitaria, y para lograrlo se propone aumentar el presupuesto de investigación, canalizar becas y sostener intercambios y acuerdos con otras universidades, entre otras acciones.

\section{Publicaciones}

Para fortalecer la calidad de las funciones de docencia y de investigación la UAPA ejecuta un amplio programa de publicaciones de libros de texto con diseño instruccional para el autoaprendizaje, guías didácticas, revistas y boletines. Dispone de su propia editora: Ediciones UAPA.

La editorial universitaria publica actualmente dos series: Documentos Institucionales, en la que se recogen Estatutos, Reglamentos, Políticas, y Manuales procedimentales; y la serie Autoaprendizaje dedicada a la publicación de libros de texto y guías didácticas.

También se publica cada semestre, la Revista Educación Superior que es el órgano de difusión de la universidad, en la que se presentan estudios e investigaciones, monografías y experiencias, tanto de la autoría de los miembros de la comunidad universitaria, como de colaboradores de otras universidades e instituciones vinculadas al mundo académico. 


\section{EXTENSIÓN RELACIONES INTERINSTITUCIONALES}

\section{Extensión}

La Universidad asume la extensión como uno de los medios que le permiten cumplir con su función social. La misma está orientada a:

- Difundir en la sociedad el quehacer institucional y los resultados de su trabajo académico y de investigación.

- Organizar y conducir programas y proyectos de asistencia social en beneficio de los sectores más necesitados.

- Contribuir en el mejoramiento de la vida cultural, artística, deportiva, científica y tecnológica del país.

Actualmente la UAPA desarrolla un novedoso Programa de Asistencia Social Comunitaria (PASC) que le ha permitido la puesta en ejecución de distintos proyectos de gran impacto y pertinencia en las comunidades pobres de su entorno. Entre ellos cabe destacar:

- Proyecto Libertad: a través del cual se brinda asistencia jurídica a personas de escasos recursos económicos.

- Proyecto Superación: centrado en la ayuda y orientación psicológica a personas maltratadas o abusadas.

- Proyecto Buscando la Luz: trabaja con la alfabetización y/o capacitación de personas adultas en edad económica mínimamente productiva.

- Este proyecto prioriza la alfabetización de trabajadores sexuales.

- Proyecto Suma: orientado a la asistencia y capacitación de micro y pequeños empresarios.

- Proyecto Comunidad Sana: contribuye con la mejoría en los niveles de salud de individuos y comunidades empobrecidas.

\section{Relaciones Interinstitucionales}

En una sociedad globalizada que avanza firmemente hacia la conformación de redes de vinculación e intercambio entre las instituciones de educación superior, es imposible mantener el aislamiento que ha caracterizado a la mayoría de las universidades dominicanas. Es de suma importancia que las IES asuman nuevas 
formas de liderazgo que le permitan el intercambio de informaciones, saberes y experiencias con distintas instituciones vinculadas al quehacer académico.

En el contexto de cambios y transformaciones que vive la República Dominicana, la Universidad debe fortalecer sus vínculos de cooperación con las empresas, asociaciones profesionales y otras organizaciones, a través de su participación en proyectos conjuntos y su oferta de servicios educativos.

La Vicerrectoría de Relaciones Internacionales y Cooperación es la responsable de planificar, ejecutar y difundir las políticas de intercambio y Cooperación Interinstitucional de la UAPA a nivel nacional e internacional. Fomenta y orienta la cooperación mutua con universidades, agencias, asociaciones y organizaciones públicas y privadas vinculadas al mundo académico. Son sus funciones y atribuciones las siguientes:

- Promover y coordinar acuerdos, convenios y alianzas con universidades e instituciones nacionales e internacionales.

- Propiciarintercambios, pasantíasyestudiosen elextranjeroparafacilitadores, participantes, egresados y personal administrativo y de servicio.

- Activar y promover la firma de convenios para la realización de investigaciones conjuntas con investigadores de universidades nacionales e internacionales.

- Formular y presentar los planes de desarrollo estratégico y operativo de la Vicerrectoría.

- Asesorar a los Recintos, Escuelasy Departamentos en la gestión de actividades de movilidad, intercambio y cooperación, nacionales e internacionales.

- Difundir y promover los asuntos internacionales de los Recintos, Escuelas y Departamentos en particular y de la Universidad en general.

- Difundir entre los miembros de la comunidad universitaria las ofertas de becas, premios y subsidios de índole internacional.

- Comunicar a la comunidad local e internacional, las actividades internacionales que se desarrollen en la Universidad tales como congresos, seminarios, investigaciones, intercambio de profesores, entre otras.

- Estimular el interés de la comunidad universitaria en particular en las diferentes actividades de internacionalización.

- Velar por la participación activa de la Universidad en los eventos académicos nacionales e internacionales.

- Mantener activos los convenios y acuerdos firmados con otras instituciones nacionales e internacionales.

- Elaborar el presupuesto anual de la Vicerrectoría. 
- Elaborar y presentar el informe anual de actividades y resultados.

- Cualquier otra competencia o función que le asigne el Rector y/o las reglamentaciones vigentes en su área.

La UAPA desarrolla múltiples actividades orientadas al fomento y fortalecimiento de las relaciones institucionales y de cooperación académica con universidades y organismos vinculados a la educación a distancia, tanto nacionales como internacionales.

Cabe destacar que en la actualidad la institución es miembro activo de las siguientes instituciones:

- La Organización Universitaria Interamericana, OUI.

- Asociación Iberoamericana de Educación a Distancia, AIESAD.

- Asociación de Televisión Educativa Iberoamericana, ATEI.

- International Council for Open and Distance Education, ICDE.

- Confederación Panamericana de Escuelas de Hotelerías y Turismo, CONPEHT.

- Asociación Dominicana de Rectores de Universidades, ADRU, ocupando actualmente la vicepresidencia.

- Asociación Dominicana para el Autoestudio y la Acreditación, ADAAC.

- Consorcio de Red de Educación a Distancia, CREAD, entre otras organizaciones.

La Universidad ha firmado acuerdos de cooperación con diversas universidades de Europa, Estados Unidos y América Latina. También muchos de sus docentes (facilitadores) se han beneficiado de oportunidades de formación que brindan diferentes organismos internacionales como la Agencia Española de Cooperación Internacional, el Banco Interamericano de Desarrollo, el Banco Mundial y la UNESCO.

$\mathrm{Al}$ favorecer las relaciones institucionales y colaborar en actividades y proyectos culturales, académicos, de desarrollo y de asistencia a la comunidad, se consolida la imagen de la institución como una Universidad solidaria, comprometida con el desarrollo del país.

\section{PLAN ESTRATÉGICO 2006 - 2007}

La planificación estratégica juega un rol fundamental como compromiso público de actuación y como mecanismo que permite que las instituciones de Educación 
Superior respondan ágilmente en un ambiente de continuo cambio, sin que las mismas tomen un camino no elegido por sus autoridades.

El Plan Estratégico de la Universidad Abierta para Adultos, UAPA contiene los lineamientos que orientarán el desarrollo de la institución en el período 2006 - 2007 y es una expresión genuina de la participación abierta y democrática de todos los sectores que conforman la comunidad universitaria.

En síntesis el Plan Estratégico es el instrumento de determinación de los objetivos que se han de alcanzar en los próximos cinco (5) años en relación a los ejes o grandes áreas predeterminadas, las estrategias precisas para alcanzar esos objetivos y las acciones y actividades específicas que concretizan esas estrategias.

En este trabajo sólo se incluyen los ejes y objetivos a lograr en el horizonte 2006 -2010 .

\section{Ejes Estratégicos}

Los ejes estratégicos constituyen las áreas o asuntos básicos de desarrollo de la misión y son a la vez la propuesta marco que ha de permitir adecuar la institución a los objetivos visionarios de su futuro ideal.

Son los grandes ámbitos aglutinadores de todas las actividades relevantes y significativas orientadas al logro de la misión y a facilitar un proceso de desarrollo institucional ordenado.

Como consecuencia de la declaración de la misión y visión de la Universidad, así como de los resultados de un análisis interno y externo de la situación actual en el que se identificaron las fortalezas, oportunidades, debilidades y amenazas de la institución, las autoridades de la UAPA identificaron seis ejes estratégicos que se presentaron, discutieron y aprobaron en los grupos de trabajo que se conformaron durante el proceso de elaboración de su Plan Estratégico. 


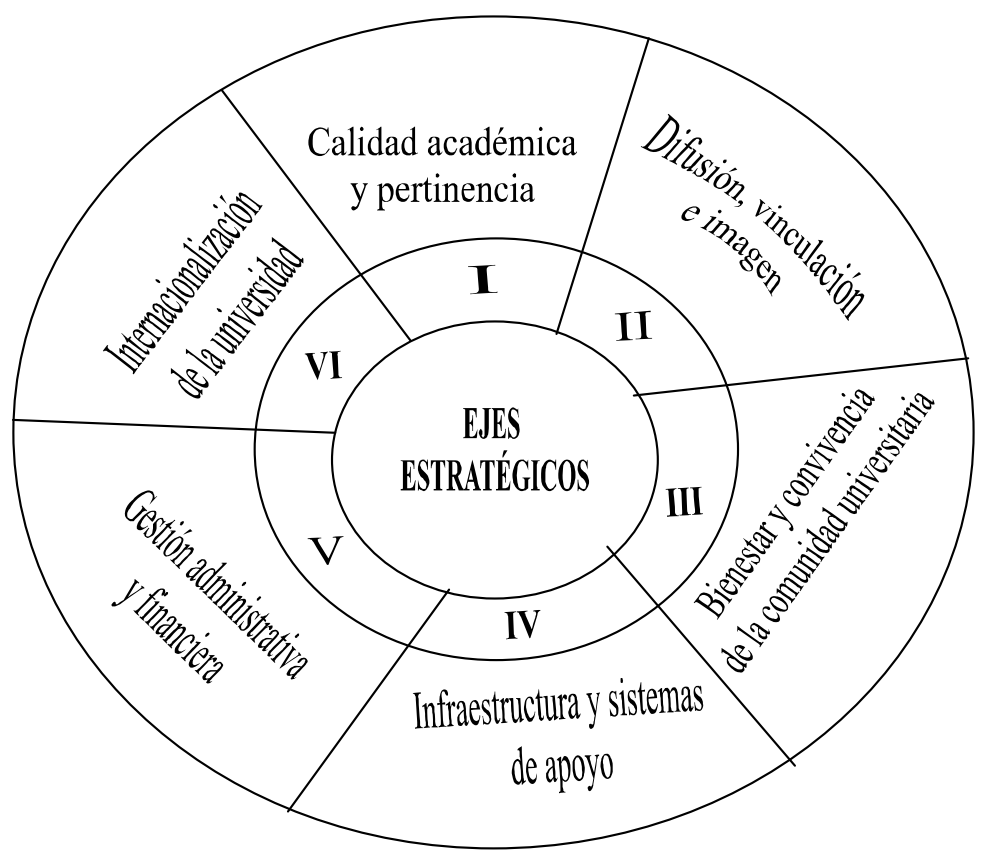

Grafico 3 - EJES ESTRATÉGICOS

\section{Objetivos Estratégicos}

El éxito del proceso de planificación requiere de la formulación de unos objetivos coherentes con la misión y valores de la Universidad y orientados a la solución de los problemas y asuntos identificados a través del análisis FODA.

Los objetivos estratégicos son las metas, finalidades y propósitos de carácter general que se pretenden conseguir en un determinado horizonte temporal. Constituyen la parte central del diseño estratégico.

Para el período 2006 - 2010 la UAPA se plantea dieciocho (18) objetivos estratégicos que se agrupan de acuerdo al eje estratégico a que corresponden.

Objetivos del Eje I: Calidad Académica y Pertinencia

- Aumentar la calidad científica, humanística, pedagógica y tecnológica de la docencia, para garantizar la formación integral de profesionales. 
- Incrementar la cantidad y calidad de la oferta de postgrado y formación permanente, asegurando su flexibilidad, atractivo y coherencia con las necesidades y demandas de la sociedad.

- Ampliar y diversificar de manera sostenible la oferta de programas académicos a nivel de grado y asegurar su adecuación a las necesidades del entorno.

- Mejorar los mecanismos de capacitación, seguimiento y fidelización de participantes.

- Desarrollar una política de investigación y transferencia de conocimientos, como soporte y referente de calidad y pertinencia de los procesos de formación y de articulación efectiva de la institución con la sociedad.

- Consolidar y asegurar la institucionalización de procesos de evaluación y autorregulación permanentes que permitan mantener a la Universidad como institución de calidad, en mejoramiento continuo.

Objetivos del Eje II: Difusión, Vinculación e Imagen

- Incrementar la oferta de servicios de extensión a la comunidad como mecanismo de aumentar y consolidar la valoración social de la Universidad.

- Ampliar e impulsar las relaciones con las empresas, organizaciones gremiales y profesionales, con los organismos públicos y con las Instituciones de Educación Superior, IES.

- Desarrollar una política de imagen corporativa y comunicación integral.

Objetivo del Eje III: Bienestar y Convivencia de la Comunidad Universitaria

- Contribuir a mejorar la calidad de vida y el entorno de trabajo del personal docente y administrativo y su identificación con los objetivos de la institución.

- Aumentar la calidad y cantidad de servicios no académicos que se ofrecen a los participantes y fomentar su participación en actividades cocurriculares en las áreas deportivas, artísticas, culturales y de preservación del medio ambiente.

- Fomentar y promover el desarrollo de relaciones sociales, constructivas entre los miembros de la comunidad universitaria, fundamentadas en el respeto por las diferencias, el diálogo, la libertad de pensamiento y la crítica constructiva. 
Objetivo del Eje IV: Infraestructura y Sistema de Apoyo

- Construir, ampliar, adecuar y/o renovar las infraestructuras de docencia, extensión, gestión y vida en los distintos recintos de la Universidad para contribuir con el mejoramiento de la calidad institucional.

- Impulsar y consolidar el desarrollo de las Tecnologías de la Información y la Comunicación (TIC's) como herramientas de apoyo y/o servicio a la docencia, la investigación, la gestión y la comunidad universitaria.

- Desarrollar mecanismos y procedimientos que aseguren la eficiencia y eficacia en la recogida sistemática y la gestión de datos de las actividades de docencia, extensión, investigación y gestión de la Universidad, que sirvan de base para la planificación y desarrollo de la Universidad.

Objetivos del Eje V: Gestión Administrativa y Financiera

- Lograr un sistema de gestión que garantice la eficiencia y eficacia en el manejo de los recursos.

- Incrementar y diversificar la generación de ingreso de la Universidad.

Objetivos del Eje VI: Internacionalización

- Incrementar y fortalecer la dimensión internacional de la Universidad en todos los ámbitos y niveles.

\section{REFERENCIAS BIBLIOGRÁFICAS}

Consejo para el desarrollo de la Ciudad y el Municipio de Santiago (2002). Plan Estratégico de Santiago. Santiago, República Dominicana: Editora de Revistas.

Farias, F. (2003). Historia Legal de Educación Superior, la Tecnología y la Ciencia Dominicana. Santo Domingo, República Dominicana: Ediciones UNICARIBE.

Perry, W. (1996). Los Sistemas de Educación a
Distancia en Europa, en Burgen, A. Metas y Proyectos de la Educación Superior: Una perspectiva Internacional. Madrid: Bocetto Gráfico, 97 - 99.

Secretaría de Estado de Educación, Ciencia y Tecnología (2003). Instituciones de Educación Superior de la República Dominicana. Directorio General. Santo Domingo: Impresel.

Universidad Abierta para Adultos (2004). Compilación de Reglamentos y Políticas 
institucionales. $\quad$ Santiago: Editora Universidad Abierta para Adultos (2004). Universitaria. Reglamento Académico. Serie Universidad Abierta para Adultos (2005). Plan Estratégico de Desarrollo 20062010. Santiago, República Dominicana. (S.E.).

Universidad Abierta para Adultos (2004). Estatutos. Serie Documentos Documentos Institucionales, 6. Santiago: Editora Búho.

Universidad Abierta para Adultos (2002). Apuntes Históricos y Memoria del año 2001. Santiago: República Dominicana.

Institucionales，5. Santiago: Editora Búho.

\section{PERFIL ACADÉMICO Y PROFESIONAL DEL AUTOR}

Ing. Fernando A. Hernández, Vicerrector de Planificación y Desarrollo de la Universidad Abierta para Adultos, UAPA. Consultor de la Secretaría de Estado de Educación Superior, Ciencia y Tecnología, SEESCyT, de la República Dominicana, en el área de Evaluación Institucional. Ing. Agrónomo por la Universidad Católica Madre y Maestra. Estudios a nivel de Maestría en Ciencias de la Educación en la Universidad Tecnológica de Santiago, UTESA. Especialista en Evaluación Institucional y Planificación Estratégica. Autor de las obras: Metodología de la Investigación en Ciencias Sociales; Investigación Documental y Comunicación Científica; Guía Práctica para la Realización y Redacción de una Monografía.

E-mail: turin.28@hotmail.com

DIRECCIÓN DEL AUTOR

Ing. Fernando Hernández

Universidad Abierta para Adultos, UAPA

Urbanización Thomen, Ave. Hispanoamericana No. 100, Código Postal No. 1238

Santiago, República Dominicana

Fecha de recepción del artículo: 24/01/07

Fecha de aceptación del artículo: 06/03/07 